\title{
Evaluación de la resistencia de unión a dentina humana de un sistema adhesivo universal con clorhexidina utilizado en modo de grabado total y autocondicionante
}

\author{
Human dentin bond strength of chlorhexidine containing universal \\ adhesive system used in total-etch and self-etch modes \\ Avaliação da resistência de união à dentina humana de \\ um sistema adesivo universal com clorexidina utilizado no \\ modo de condicionamento total e autocondicionante
}

\author{
Romina Tessore ${ }^{1}$ ORCID: 0000-0001-6259-042X \\ Camila Silveira' ORCID: 0000-0003-4631-9116 \\ Priscila Vázquez ${ }^{1}$ ORCID: 0000-0002-3326-2547 \\ Matías Mederos ${ }^{1}$ ORCID: 0000-0002-1561-2283 \\ Andrés García ${ }^{1}$ ORCID: 0000-0001-6733-4484 \\ Carlos Enrique Cuevas-Suarez² ORCID: 0000-0002-2759-8984 \\ Guillermo Grazioli ${ }^{1}$ ORCID: 0000-0001-9969-3780
}

DOI: 10.22592/ode2020n35a4

\section{Resumen}

Objetivos: Evaluar la resistencia de unión a la microtracción en dentina humana de un sistema adhesivo universal con clorhexidina en su composición, en modo de grabado y lavado en 2 pasos, y en modo de autograbado.

Metodología: 20 terceros molares divididos aleatoriamente en 4 grupos según el tipo de sistema adhesivo utilizado (Single Bond Universal ${ }^{\oplus}, 3$ MESPE y Peak Universal Bond ${ }^{\oplus}$, Ultradent Products) y modo de uso (grabado total y autograbado). Se confeccionaron cuerpos de prueba sometidos al ensayo de microtracción utilizando una máquina de ensayos universales.

Resultados: No se encontraron diferencias estadísticamente significativas entre los cuatro grupos estudiados.

Conclusiones: La resistencia de unión de una resina compuesta a dentina humana no fue afectada por el uso de un sistema adhesivo universal que contiene clorhexidina en su composición aplicado en los modos de grabado total y autograbado.

Palabras clave: clorhexidina, microtracción, metaloproteinasas, capa híbrida.

Cátedra de Materiales Dentales, Facultad de Odontología, Universidad de la República, Uruguay.

Laboratorio de Materiales Dentales, Área académica de Odontología, Universidad Autonoma del Estado de Hidalgo, México.

Fecha de recibido: 20/09/2019 - Fecha de aceptado: 30/01/2020 


\section{Abstract}

Objectives: To evaluate the microtensile bond strength to human dentin of chlorhexidine containing universal adhesive system applied in the total-etch and selfetch modes.

Methods: Twenty third molars were randomly divided into four groups according to the universal adhesive system (Single Bond Universal ${ }^{\bullet}$, 3M ESPE and Peak Universal Bond ${ }^{\oplus}$, Ultradent) and application mode used (total-etch and self-etch). Specimens were prepared and subjected to a microtensile bond strength using a MTS SANS universal testing machine.

Results: No statistically significant differences were found in the microtensile bond strength between the four groups studied.

Conclusions: The bond strength of a composite resin to human dentin was not affected by the use of a universal adhesive system with chlorhexidine in its composition applied in the total-etch and self-etch modes.

Keywords: chlorhexidine, microtensile, metalloproteinases, hybrid layer.

\section{Introducción}

Desde la introducción del grabado ácido por Buonocore en el año 1955, se ha visto un aumento en la demanda de restauraciones adhesivas estéticas ${ }^{(1)}$, determinando el desarrollo de diferentes estrategias adhesivas ${ }^{(2)}$ en base a la conservación/integración del barillo dentinario (smear layer) ${ }^{(3-4)}$ : eliminación del barro dentinario o técnica de grabado y lavado (etch and rinse) $)^{(5-8)}$, y autograbado (self-etch) que integra al barrillo a la interfase adhesiva. Ambas técnicas adhesivas se comportan de forma diferente a nivel de la dentina. En cuanto a la técnica

\section{Resumo}

Objetivos: Avaliar a resistência de união á microtração na dentina humana de um sistema adesivo universal com clorexidina na sua composição, no modo de condicionamento total em dois passos e no modo autocondicionante.

Metodologia: 20 terceiros molares foram divididos aleatoriamente em 4 grupos, de acordo com o tipo de sistema adesivo utilizado (Single Bond Universal ${ }^{\oplus}$, 3MESPE e Peak Universal Bond ${ }^{\circledR}$, Ultradent Products) e modo de uso (condicionamento total e autocondicionante). Os corpos de prova criados foram submetidos ao teste de microtração utilizando uma máquina de ensaios universal.

Resultados: Não foram encontradas diferenças estatisticamente significativas entre os quatro grupos estudados.

Conclusóes: A resistência de união da resina composta à dentina humana não foi afetada pelo uso de um sistema adesivo universal contendo clorexidina em sua composição, aplicado nos modos de condicionamento total ou autocondicionamento.

Palavras-chave: clorexidina, microtração, metaloproteinases, camada híbrida.

de grabar y lavar, si bien es la más utilizada, su efectividad está discutida, dado que los monómeros del adhesivo no siempre logran humectar todas las superficies grabadas, dejando una capa híbrida con espacios vacíos y fibras colágenas sin sostén ${ }^{(9)}$. Por otra parte, la técnica de autograbado plantea la capacidad de remover minerales de la capa superficial del barro y reemplazarlos simultáneamente por monómeros de resina ${ }^{(10,11)}$. En el proceso de adhesión se reconoce un fenómeno llamado nano filtración que se produce gracias a la presencia de espacios vacíos de tamaño nanométrico que incorporaran fluidos desde la pulpa dental hacia la su- 
perficie externa de la dentina ${ }^{(14)}$. Estos espacios se generan porque los monómeros resinosos no llegan a infiltrar completamente los espacios generados entre las fibras colágenas resultando en una zona de dentina desmineralizada debajo de la capa híbrida, pudiendo originar con el tiempo una degradación por hidrólisis de la unión resina-dentina ${ }^{(11,15)}$. Además, las fibras colágenas no protegidas (no rodeadas de resina o dentina) pueden ser degradadas por enzimas proteolíticas endógenas que se encuentran en la dentina, llamadas metaloproteinasas (MMP) de la matriz. Estas enzimas son responsables de la degradación de la matriz extracelular en diferentes procesos fisiológicos ${ }^{(11,15)}$. A nivel de la capa híbrida, estas enzimas son responsables de una disminución de la resistencia de unión de los materiales resinosos a la dentina a largo plazo ${ }^{(16)}$.

En la búsqueda de contrarrestar el efecto de las MMP, se ha planteado el uso de diferentes agentes como: riboflavina ${ }^{(17)}$, extractos naturales $^{(17)}$ y clorhexidina ${ }^{(11,15,17)}$. La clorhexidina es un compuesto químico sintético que posee destacables propiedades antibacterianas, es ampliamente utilizado en diversos procedimientos médicos, quirúrgicos y odontológicos ${ }^{(11,15)}$. En la odontología restauradora la clorhexidina fue inicialmente introducida como desinfectante de las cavidades antes de la aplicación de materiales restauradores, con el objetivo de eliminar las bacterias que podrían irritar la pulpa dental $^{(11,15)}$. Además, se ha constatado un efecto inhibitorio contra actividad proteolítica de las MMPs -2, -8 y -9,contribuyendo a una mayor longevidad de la unión resina-dentina ${ }^{(11,15)}$.

Actualmente, en búsqueda de simplificar la toma de decisión y técnica clínica, se encuentran en el mercado adhesivos denominados Universales, debido a su versatilidad (en poder aplicarse como grabado y lavado o en autograbado) y también por su afinidad con múltiples sustratos (dentarios, metales y/o cerámicas) ${ }^{(3,5,14)}$.

Además, se encuentran disponibles en el mercado sistemas adhesivos con clorhexidina en su composición, lo que podría colaborar con la estabilidad adhesiva al disminuir la degradación de la articulación adhesiva, aumentando la durabilidad y éxito de las restauraciones plásticas adhesivas $^{(11,15)}$ La reciente introducción al mercado de este adhesivo universal con clorhexidina en su composición, genera interrogantes en cuanto a su eficacia, sus ventajas y propiedades a los consumidores (odontólogos) ya que no existe suficiente evidencia de que el agregado de clorhexidina dentro de su composición no afecte su desempeño.

En este contexto, el presente trabajo tiene como objetivo comparar la resistencia de unión a dentina de un nuevo sistema adhesivo comercial universal con clorhexidina en su composición, y comparar los valores obtenidos con un sistema adhesivo comercial universal sin clorhexidina en su composición en estrategias de grabado y lavado, y en autograbado.

Las hipótesis planteadas son que el sistema adhesivo con clorhexidina en su composición presentará valores similares de resistencia adhesiva comparado con el sistema adhesivo sin clorhexidina, además de que ambos no presentarán diferencias estadísticamente significativas entre la técnica de grabado y lavado con la de autograbado.

\section{Objetivo}

Evaluar la resistencia de unión en dentina humana de un sistema adhesivo universal con clorhexidina en su composición, y comparar los valores de resistencia de unión obtenidos con los de un sistema adhesivo universal sin clorhexidina en los modos de grabado y lavado y autograbado.

\section{Metodología}

\section{Obtención de las muestras}

La muestra fue constituida por 20 terceros molares sanos sin importar el estado de desarrollo radicular. Las piezas dentarias se obtuvieron 
del servicio de block quirúrgico de Facultad de Odontología. Los pacientes brindaron consentimiento informado escrito de donación para la investigación (Protocolo aprobado por el comité de ética de la Facultad de Odontología UdelaR - No. De Exp. 278/16), donde se esclareció que la indicación de extracción fue por motivos ajenos a esta investigación (ej. ortodoncia). El procedimiento de recolección, limpieza y almacenamiento de los dientes se encuentra protocolizado por el Banco de Dientes Humanos de la Facultad de Odontología (Exp. 091100000933-11, Consejo de la Facultad de Odontología- UdelaR).

Una vez extraídos, los órganos dentarios se almacenaron en Cloramina T 0,5\% por 7 días y posteriormente en agua destilada a una temperatura de $3^{\circ}$ a $5^{\circ} \mathrm{C}$ hasta el momento del estudio, por no más de 3 meses.

La muestra se dividió aleatoriamente en cuatro grupos con 5 molares cada uno:

Grupo 1: Adhesivo Universal, Single Bond Universal (3M ESPE; ST PAUL, MN, USA), con técnica de grabado y lavado (SBU TE).

Grupo 2: Adhesivo Universal, Single Bond Universal (3M ESPE; ST PAUL, MN, USA), con técnica de autograbado (SBU SE).

Grupo 3: Adhesivo Universal con clorhexidina al $0,2 \%$ en su composición, Peak Universal Bond (Ultradent Products; South Jordan, Utah, USA), con técnica de grabado y lavado (PBU TE).

Grupo 4: Adhesivo Universal con clorhexidina al $0,2 \%$ en su composición, Peak Universal Bond (Ultradent Products; South Jordan, Utah, USA), con técnica de autograbado (PBU SE).

\section{Preparación de las muestras para el ensayo de microtracción}

Cada pieza dentaria fue desgastada transversalmente, utilizando una recortadora con irriga- ción, eliminando el esmalte de la cara oclusal, exponiendo una superficie plana de dentina coronaria, sin exposición pulpar. Para la estandarización del barro dentinario, la dentina coronaria fue posteriormente desgastada con una lija de carburo de silicio con granulometría 600 realizando suaves movimientos en forma de ocho e irrigación abundante. Sobre la superficie dentinaria expuesta, se realizó el tratamiento adhesivo correspondiente según el grupo al que pertenecía la muestra, siguiendo las indicaciones del fabricante (Tabla 1). El uso de los adhesivos seleccionados corresponde a las indicaciones del fabricante. Luego de los procedimientos adhesivos, se creó un bloque de $4 \mathrm{~mm}$ de resina compuesta $3 \mathrm{M}^{\text {Filtek }}{ }^{\mathrm{TM}} \mathrm{Z} 250 \mathrm{XT}$ (3M ESPE; ST PAUL, MN, USA), manipulando la misma según lo indicado por el fabricante. La polimerización se realizó utilizando una unidad de fotocurado Optilight Max (Gnatus; Ribeirao Preto, Brasil). La irradiancia de la unidad de fotocurado fue testeada con un radiómetro Bluephase Meter (Ivoclar Vivadent; Schaan, Linchenstein, Alemania) que aseguró su correcto funcionamiento. (Ver tabla 1)

Luego de ser realizadas, las muestras se almacenaron por 24 horas en agua destilada a $37^{\circ} \mathrm{C}$. Pasado ese periodo, los dientes fueron seccionados utilizando una microcortadora GellingsHamco (Hamco Machines INC.; Rochester, NY, USA), para obtener bastones de $1 \mathrm{~mm}^{2}$ de sección (Fig. 1), (aproximadamente 8 bastones por pieza dentaria) los cuales fueron mitad dentina y mitad resina compuesta. Para el ensayo de microtracción, fueron seleccionados 5 bastones obtenidos de la región central de cada uno de los dientes $(\mathrm{n}=25)$. 
Tabla 1. Nombre, composición y procedimiento de aplicación de los materiales utilizados en el presente estudio.

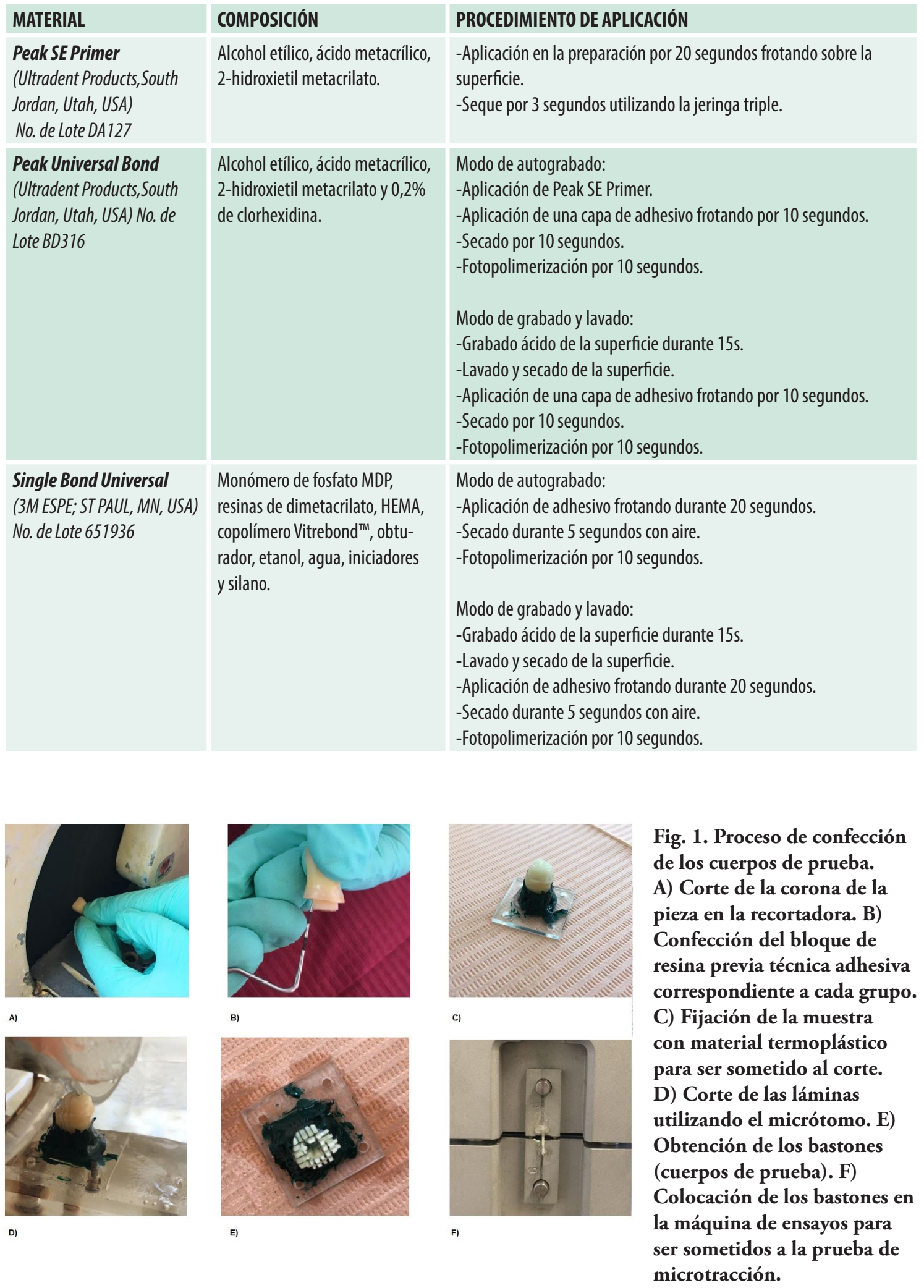


Para este ensayo se utilizó un dispositivo que consiste en 2 placas de acero inoxidable que se desplazan entre ellas. Cada bastón fue pegado de ambos extremos con cianoactilato a dichas placas, dejando la interfaz diente-restauración libre entre ambas placas. De esta manera, la fuerza de tracción se transmitió al bastón ${ }^{(18)}$. El ensayo de microtracción se realizó utilizando una máquina de ensayos universales MTS SANS CMT 2000 5KN (MTS Systems Corporation; Shanghai China). Los cuerpos de prueba fueron sometidos a ensayos de microtracción siguiendo la norma ISO 11405 (Fig. 2) ${ }^{(19)}$.Una vez fracturados, los cuerpos de prueba fueron analizados en el microscopio a una magnificación de 40x para clasificar el tipo de fractura en adhesiva, cohesiva en dentina y cohesiva en resina.

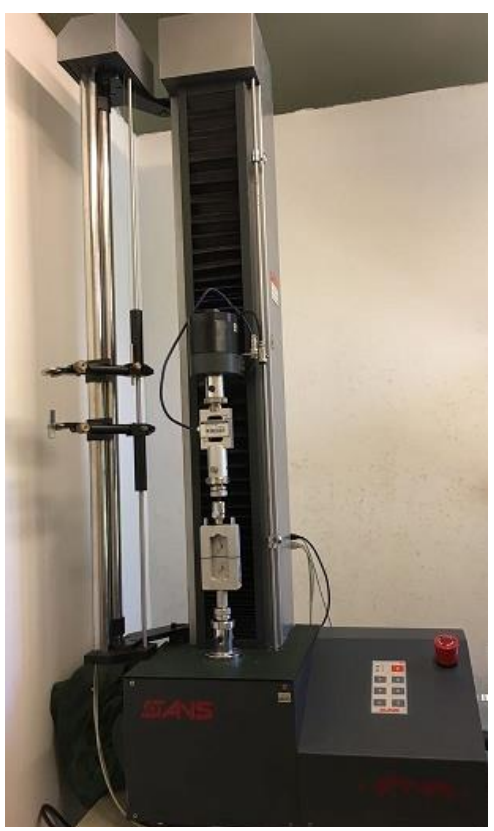

Fig. 2.

Máquina de ensayos universales (MTS SANS CMT 2000 $5 \mathrm{KN})$.

\section{Análisis estadístico}

Los resultados obtenidos fueron analizados realizando un test de ANOVA de dos vías utilizando los factores de material (Peak Universal bond o Single bond Universal) y modo (grabado y lavado o autocondicionamiento) para comparar la resistencia de unión entre los distintos siste- mas adhesivos. La variabilidad en la frecuencia de la distribución de los tipos de fractura fue analizada realizando la prueba de Chi-cuadrada. Un nivel de significancia de $\alpha=0,05$ fue utilizado para todos los análisis.

\section{Resultados}

Los valores (media y desvío estándar) de la resistencia de unión en microtracción en dentina se muestran en la Figura 3. De acuerdo con el gráfico, el sistema adhesivo Peak Universal Bond (Ultradent) con técnica de autograbado fue el que obtuvo mayores valores de resistencia de unión, mientras que el sistema de autograbado Single Bond Universal (3M ESPE) obtuvo en promedio los menores valores de resistencia adhesiva. Sin embargo, no se observaron diferencias estadísticamente significativas entre ninguno de los cuatro grupos estudiados luego de realizar un ANOVA de dos vías (factor material $\mathrm{p}=0,537$, factor modo $\mathrm{p}=0,864$, interacción de factores $\mathrm{p}=0,27)$.

En cuanto al tipo de fallas, todos los grupos presentaron un predominio de fallas adhesivas, y en menor proporción fallas cohesivas en dentina. Sin embargo, en el grupo que se utilizó como sistema adhesivo el Peak Universal Bond, en modo autocondicionante, presentó mayor cantidad de fallas cohesivas en dentina (Fig. 4). No fueron observadas fallas mixtas. La variabilidad en las frecuencias de los tipos de falla entre los diferentes grupos no fue estadísticamente significativa $(\mathrm{p}=0,121)$.

\section{Discusión}

En el presente estudio no se encontraron diferencias estadísticamente significativas en los valores de resistencia de unión entre los dos sistemas adhesivos utilizados, así como también no fueron identificadas diferencias estadísticamente significativas entre los modos de uso de grabado y lavado (etch and rinse) y autograbado 


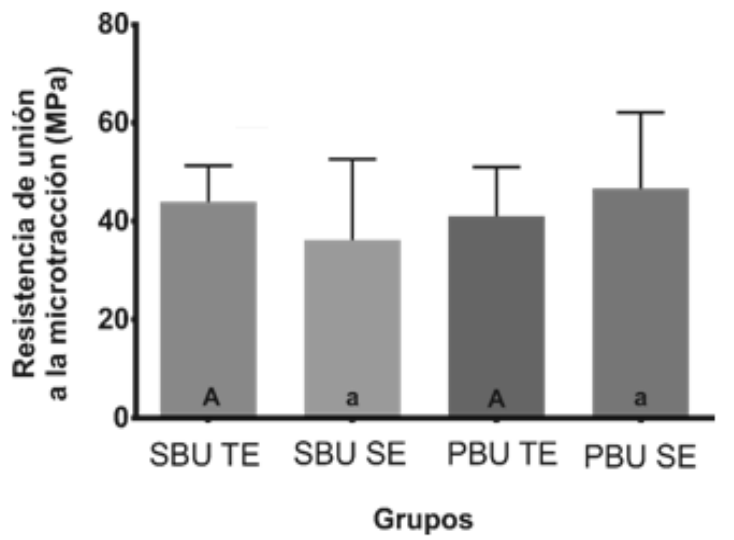

\begin{tabular}{|c|c|c|c|c|}
\hline & SBU TE & SBU SE & PBU TE & PBU SE \\
\hline Media & 44,0 & 36,2 & 41,0 & 46,7 \\
\hline DE & 7,3 & 16,4 & 10,0 & 16,4 \\
\hline
\end{tabular}

Fig. 3. Gráfico: Resistencia de unión a la microtracción en función del sistema adhesivo (Single Bond Universal - SBU o Peak Bond Universal - PBU) el modo de uso (grabado y lavado - TE o autograbado - SE). Las letras mayúsculas iguales indican ausencia de diferencias entre los sistemas adhesivos usados en el modo de grabado y lavado. Las letras minúsculas iguales indican la ausencia de diferencias entre los adhesivos usados en el modo autograbado. Cuadro: Media aritmética y desvío estándar de la resistencia de unión a la microtracción.

Adhesiva Cohesiva en dentina Cohesiva en resina

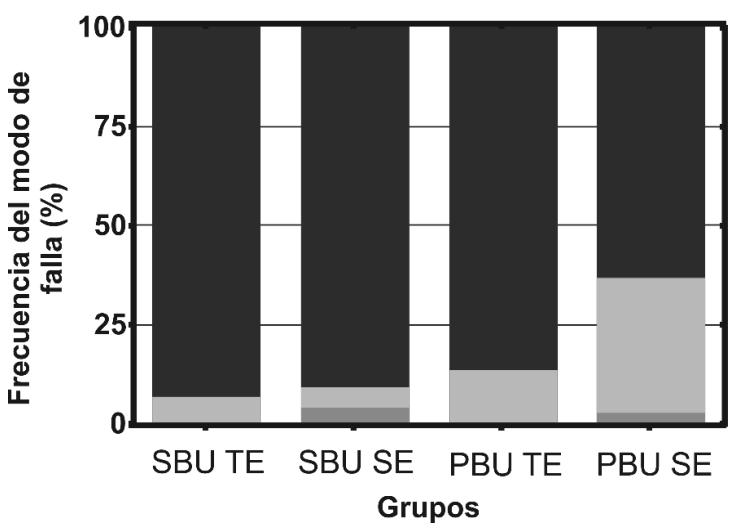

Fig. 4. Análisis del modo de falla de los grupos evaluados. (self-etch). Considerando esto, nuestra hipótesis de trabajo puede ser aceptada.

Estos resultados coincidieron con diversos estudios que demuestran que hoy en día gracias al avance tecnológico y científico se logran valores estadísticamente similares utilizando sistemas adhesivos de grabado y lavado y de autograbado. Tradicionalmente, en algunos artículos se defendía la superioridad de los sistemas de grabado y lavado frente al uso de autograbado en dentina ${ }^{(4,5,13)}$. Actualmente existen estudios que demuestran que utilizando la técnica de autograbado en dentina se obtienen valores de adhesión superiores a la técnica tradicional de grabado y lavado ${ }^{(12)}$.

Se ha demostrado que valores de resistencia de unión a dentina obtenidos por ensayos de microtracción utilizando sistemas adhesivos de autograbado en dos pasos y grabado total, demostraron valores estadísticamente semejantes, a excepción del sistema autograbado en un paso que se aleja de los valores ${ }^{(8)}$. En la literatura se relata que los sistemas autograbado en un paso, no tienen buenos resultados en cuanto a la resistencia de unión posiblemente debido a la combinación de ácidos y monómeros hidrofílicos e hidrofóbicos en una solución única ${ }^{(22)}$. Un estudio concluyó que, independientemente del sustrato en que se apliquen los sistemas (dentina superficial o profunda), la resistencia de unión fue mayor en el sistema autograbador de dos pasos en relación al de un paso o al sistema de grabado total convencional ${ }^{(22)}$. Además, se evidencia que los sistemas adhesivos de autograbado presentan menor confiabilidad en cuanto a resistencia de unión y nanofiltración comparados con los sistemas de grabado y lavado de tres pasos, los cuales se mantienen como estándar de oro para estudios comparativos ${ }^{(1)}$. Entre los sistemas de autograbado, los de dos pasos, tienen mejor desempeño clínico y de laboratorio que los de un paso. A su vez, los sistemas adhesivos de autograbado presentan mejor desempeño en adhesión a dentina que a esmalte $^{(23)}$. 
Los adhesivos universales están indicados para ser utilizados con la técnica de grabado total y de autograbado, y de acuerdo con el fabricante, tienen la misma eficacia en cualquiera de los modos utilizados. Estudios recientes han demostrado que, durante el grabado ácido, las enzimas metaloproteinasas de matriz (MMP) se liberan de la dentina y pueden degradar la capa híbrida. Con el fin de controlar el efecto de las MMP se ha estudiado el uso de diferentes agentes, entre ellos la clorhexidina. Este compuesto presenta propiedades antibacterianas que inhiben la actividad proteolítica de las MMP a muy bajas concentraciones, por lo tanto, se inhibe la degradación de la capa híbrida, logrando de esta forma una mayor longevidad de la unión resina-dentina ${ }^{(11,15)}$.

En este estudio se comparó la eficacia adhesiva de dos sistemas adhesivos universales, de los cuales, uno de ellos contiene clorhexidina en su composición. Los resultados, revelaron que el uso de clorhexidina no influye en la resistencia de unión ${ }^{(24)}$. Estos resultados sugieren que es posible utilizar sistemas adhesivos que contienen clorhexidina al $0,2 \%$ en su composición. A pesar de este resultado, es importante mencionar que el grupo que utilizó como sistema adhesivo el Peak Universal Bond en modo autocondicionante (self-etch), presentó mayor cantidad de fallas cohesivas en dentina, dejando en evidencia una posible mayor interacción de este sistema adhesivo con el sustrato dentario respecto a los otros grupos.

A pesar de que el uso de clorhexidina no incide directamente en la resistencia de unión inmediata, es posible que, gracias a su rol como inhibidor de las MMP, los resultados a largo plazo puedan ser diferentes. En otra revisión, se estudia la incorporación de clorhexidina en el primer de algunos adhesivos de autograbado en dos pasos, y se observó que, durante el protocolo de adhesión, permite inhibir la acción de las MMP hasta cierto punto y no tiene efectos adversos en la resistencia de unión inmediata al sustrato dentinario ${ }^{(23)}$.
Por ende, podemos resumir que tanto las revisiones anteriormente mencionadas como el estudio presente, coinciden en que la clorhexidina juega un papel importante en cuanto a su acción inhibitoria de las MMP sin generar efectos adversos en la resistencia de unión independientemente del sistema adhesivo utilizado, siendo que la elección de la técnica de grabado y lavado o autograbado no presenta diferencias en su resistencia de unión.

A modo de poder ampliar los conocimientos ya generados con este estudio in vitro, los autores recomiendan realizar otros estudios in vitro a mediano y largo plazo para monitorizar la presencia de clorhexidina en la interfaz adhesiva y su efecto en la fuerza de unión. Por otro lado, una vez agotados los estudios in vitro, se recomiendan ensayos clínicos randomizados que puedan corroborar el desempeño clínico de estos sistemas adhesivos con el fin de constatar la longevidad de las restauraciones y la interfaz de unión obtenida con estos distintos sistemas adhesivos. Debemos destacar que este estudio presentó limitaciones en cuanto a la cantidad de muestras estudiadas, ya que un n mayor podría disminuir la desviación estándar, permitiendo reconocer alguna diferencia estadísticamente significativa. Además, debemos considerar que simplemente la resistencia de unión no brinda un conocimiento integral sobre el desempeño de un sistema adhesivo, existiendo otras metodologías in vitro que podrían ser ejecutadas como microfiltración, ensayos de resistencia de unión luego de un envejecimiento, y análisis de microscopia electrónica de las interfases adhesivas, entre otros, que permitan tener un panorama más completo del desempeño laboratorial de un sistema adhesivo. Por otro lado, como fortalezas de este estudio, el cumplimiento metodológico de la normativa ISO vigente, la estandarización de las muestras y los protocolos, y el uso del equipamiento específico, junto con la corroboración de los datos obtenidos que concuerdan con la literatura existente, sustentan la fiabilidad de los datos publicados. 


\section{Conclusiones}

A pesar de las limitaciones de este estudio, es posible concluir que el desempeño de los sistemas adhesivos universales no depende del modo de uso en que es utilizado en la dentina, del mismo modo, fue posible observar que la incorporación de clorhexidina a un sistema adhesivo universal no afectó significativamente su desempeño.

Considerando que la clorhexidina cumple un papel muy importante al inhibir las MMP, es posible que el uso de este tipo de adhesivos evite la degradación de la capa híbrida y, por consiguiente, la longevidad de la unión resinadentina sea aumentada. Futuros estudios deben ser realizados para corroborar esto.

\section{Agradecimientos}

El presente trabajo fue financiado por el Programa de Apoyo para la Investigación Estudiantil (PAIE) de la Comisión Sectorial de Investigación Científica (CSIC).

\section{Conflicto interés}

Los autores declaran no tener algún conflicto de interés.

\section{Contribución de autoría}

1. Concepción y diseño del estudio

2. Adquisición de datos

3. Análisis de datos

4. Discusión de los resultados

5. Redacción del manuscrito

6. Aprobación de la versión final del manuscrito

RT ha contribuido en 1, 2, 4, 5 .

CS ha contribuido en 1, 2, 4, 5 .

PV ha contribuido en 1, 2, 4, 5 .

MM ha contribuido en 4, 5 .

AG ha contribuido en 4, 5.

CECS ha contribuido en 3, 4.

GG ha contribuido en 1, 3, 4, 6 .

\section{Referencias}

1. Correa MB, Peres MA, Peres KG, Horta BL, Barros AD, Demarco FF. Amalgam or composite resin? Factors influencing the choice of restorative material. J Dent. 2012; 40 (9): 703-10.

2. Bedran-Russo A, Leme-Kraus AA, Vidal CMP, Teixeira EC. An Overview of Dental Adhesive Systems and the Dynamic Tooth-Adhesive Interface. Dent Clin North Am. 2017; 61 (4): 713-731.

3. Sofan E, Sofan A, Palaia G, Tenore G, Romeo U, Migliau G. Classification review of dental adhesive systems: from the IV generation to the universal type. Ann Stomatol (Roma). 2017; 8 (1):1-17.

4. Tsujimoto A, Iwasa M, Shimamura Y, Murayama R, Takamizawa T, Miyazaki M. Enamel bonding of single-step self-etch adhesives: Influence of surface energy characteristics. J Dent. 2010; 38 (2):123-30.

5. Mandri MN, Aguirre Grabre de Prieto A, Zamudio ME. Sistemas adhesivos en Odontología Restauradora. Odon. 2015; 17 (26): 50-6.

6. Van Meerbeek B, Peumans M, Poitevin A, Mine A, Van Ende A, Neves A, De Munck J. Relationship between bond-strength tests and clinical outcomes. Dent Mater. 2010; 26 (2): 100-21.

7. Atash Biz Yeganeh L, Seyed Tabai E, MohammadiBasir M. Bonding Durability of Four Adhesive Systems. J Dent (Tehran). 2015; 12 (8): 563-70.

8. Dourado Loguercio A, Reis A. Sistemas Adhesivos. RevOperDent y BiomaterDent y Biomater. 2006; 1 (2): 13-28

9. Hernández JM. Aspectos prácticos de la adhesión a dentina Practicalaspects in dentinaladhesion. Av. Odontoestomatol. 2004; 20 (1): 19-32.

10. Ceballos L, Camejo DG, Victoria Fuentes M, Osorio R, Toledano M, Carvalho RM, Pashley $\mathrm{DH}$. Microtensile bond strength of total-etch and self-etching adhesives to caries-affected dentine. J Dent. 2003; 31 (7): 469-77.

11. Carrilho MR, Geraldeli S, Tay F, de Goes MF, Carvalho RM, Tjäderhane L, Reis AF, Hebling J, Mazzoni A, Breschi L, Pashley. In vivo preservation of the hybrid layer by chlorhexidine. J Dent Res. 2007; 86 (6): 529-33. 
12. Oliveira SSA, Pugach MK, Hilton JF, Watanabe LG, Marshall SJ, Marshall GW. The influence of the dentin smear layer on adhesion: A selfetching primer vs. a total-etch system. Dent Mater. 2003; 19 (8): 758-67.

13. Scherrer SS, Cesar PF, Swain M V. Direct comparison of the bond strength results of the different test methods: a critical literature review. Dent Mater. 2010; 26 (2): e78-93.

14. Bader Mattar M, Ibáñez Musalem M. Evaluación de la interfase adhesiva obtenida en restauraciones de resina compuesta realizadas con un sistema adhesivo universal utilizado con y sin grabado ácido previo. Rev Clínica Periodoncia, Implantol y Rehabil Oral. 2014; 7 (3): 115-22.

15. Pomacóndor-Hernández C. Papel de la clorhexidina en la odontología restauradora. Odontol San Maquina. 2010; 13 (2): 46-9.

16. Tekçe N, Tuncer S, Demirci M, Balci S. Do matrix metalloproteinase inhibitors improve the bond durability of universal dental adhesives? Scanning. 2016; 38 (6): 535-44.

17. Vola J. Influencia de los inhibidores de las metaloproteinasas, agentes reticuladores y remineralizaciónbiomimética en la longevidad de la unión adhesiva. Actas Odon. 2014; 11 (2): $10-21$.

18. Pashley DH, Carvalho RM, Sano H, Nakajima M, Yoshiyama M, Shono Y, Fernandes CA, Tay
F. The microtensilebond test: a review. J Adhes Dent. 1999; 1 (4): 299-309.

19. International Organization for Standarization. ISO 4049:2009 Dentistry Polymer based restorative materials. 2009.

20. Leite ML de A e. S, Costa CA de S, Duarte RM, de Andrade AKM, Soares DG. Bond strength and cytotoxicity of a universal adhesive according to the hybridization strategies to dentin. Braz Dent J. 2018; 29 (1): 68-75.

21. Muñoz MA, Luque I, Hass V, Reis A, Loguercio $\mathrm{AD}$, Bombarda NHC. Immediate bonding properties of universal adhesives to dentine. J Dent. 2013; 41 (5): 404-11.

22. Sanchez F, Osorio R, Toledano M. Control del colapso del colágeno: sistemas autograbadores. Av. Odontoestomatol. 2004; 20 (4): 175-83.

23. Parra Lozada M, Rayo HG. Sistemas adhesivos autograbadores, resistencia de unión y nanofiltración: una revisión self-etchingadhesivesystems, bond strength and nanofiltration: a review. Rev Fac Odontol Univ Antioquia. 2012; 24 (24): 133-150.

24. Roopa D ARS, D R. por Roopa D; Rao A. Comparative Evaluation of the Bonding Efficacy of Seventh Generation Bonding Agent and Peak Universal Bond: An In-Vitro Study. JBR J Interdiscip Med Dent Sci. 2015; 03 (02): 1-4.

Guillermo Grazioli: ggrazioli@gmail.com 